\title{
Research on Fusion Model Characteristics of Wind Farm
}

\author{
Qianli Ma, Xuemin Zhang \\ Tsinghua University, China \\ *Maqianlithu@126.com
}

\begin{abstract}
Keywords: wind farm characteristics;simplified model;Short circuit fault;SVR algorithm
\end{abstract}
\begin{abstract}
Wind farm characteristics is the basis of the research on wind power grid operation and control. The main purpose of this paper is to establish a simplified structure, which is a high security, easy maintenance wind farm model. This model can calculate accurate response in the grid disturbance. The idea of modeling is mainly based on the feature fusion method, which is extracted from the detailed model and calculated by SVR algorithm to get parameters. The accuracy of the simplified model analysised by comparing the transient response during grid disturbance.
\end{abstract}

\section{Introduction}

China is the largest developing country in the world, and also the largest energy consumption country and energy producer. The shortage of fossil energy makes China's energy transformation imperative. Since twenty-first Century, China's wind power industry has developed rapidly, with more than $10 \%$ annual growth in installed capacity increased. The distribution of wind energy in China is mainly concentrated in the northeast, the north, the northwest, the Qinghai Tibet Plateau and the southeast coastal areas. These areas are affected by geographical factors or monsoon. They have a good wind power base. The wind speed exceeds $3 \mathrm{~m} / \mathrm{s}$ for over 4000 hours a year. The annual average wind speed in some areas can reach $6-7 \mathrm{~m} / \mathrm{s}$. It is a concentrated area of wind farms in China.

Wind power has many advantages. (1) wind power is environmentally friendly, with no pollutant emission. (2) wind power construction cost and operation cost is low. Wind farms can mostly be built in about 1 years. The installed capacity can be flexibly adjusted according to needs and funds. The automation degree of modern wind farms is very high. It can achieve stable operation without human being. It only needs regular maintenance, and the running cost is about 2-3\% of initial investment. The development of wind power generation is also restricted by many factors, mainly as follows: (1) the wind speed has strong volatility and randomness. (2) the development of energy storage technology has an important impact on wind power. (3) wind power will cause a certain impact on the ecological, such as noise pollution.

The detailed model of the wind farm is very complex, and many papers set up the simplified model of the wind field from various angles. It is easier to calculate the precision within the range of accuracy. The modeling of double- fed induction generator is made by document [1]. According to the characteristic vectors of fan running point, the clustering is equivalent. The clustering is mainly based on the $\mathrm{K}$ - means clustering algorithm, and the result is corrected by means of $\mathrm{C}$ - means clustering algorithm. The number of families of $\mathrm{k}$ - mean clustering is raised. The disadvantage is that the result of k- mean clustering is unstable and has a certain randomness. Document [2] explores the influence of pitch angle on operation point during fan operation, and takes impeller angle and wind speed as two indicators to determine the running state of the unit, and gives control strategy for controlling the pitch angle. Document [5] takes into account the time constant of dynamic response, classifying by using speed instead of wind speed. Clustering is still using k- means clustering algorithm. After classifying, the weight of each class is generated, and the equivalent model of $\mathrm{N}$ typhoon machine is obtained.

The above literature discusses the research background and determine several equivalent model of wind farm, this paper emphasizes on Modeling of wind farm using characteristics of the integration of ideas, and to learn from each other, first through a detailed model of wind farm, set training 
characteristics as a fusion algorithm to extract a set of data, calculate the parameters by SVR, the equivalent model is given. And then examine the accuracy of the equivalent model of power grid in a variety of disturbances, and the power system simulation test of a small, and treat problems put forward.

\section{A Summary of The Principles}

\subsection{Wind Turbine Generator}

Wind power generators are divided into synchronous motors and asynchronous motors. The direct drive wind turbine generator is a widely used synchronous generator. It has many advantages, such as long life, low failure rate and simple maintenance. In addition, With high power generation efficiency, it is very suitable for low speed operation. when the grid voltage drop, direct drive type wind machine can run continuously in a large range.

\subsection{Wind Electric Field Model}

The wind farm model is divided into two types, the mechanism modeling and the non mechanism modeling. The mechanism modeling is a detailed model constructed by the physical model and the mathematical formula. the advantages of this modeling approach is that there is a clear mathematical relationship between the inputs and outputs. However, there are some complex factors in wind farm modeling that cannot be calculated easily, which would cause errors in the outputs. In non mechanism modeling, the system is like a black box. A mathmatical formula is analysised to match the input data and output data as close as possible.

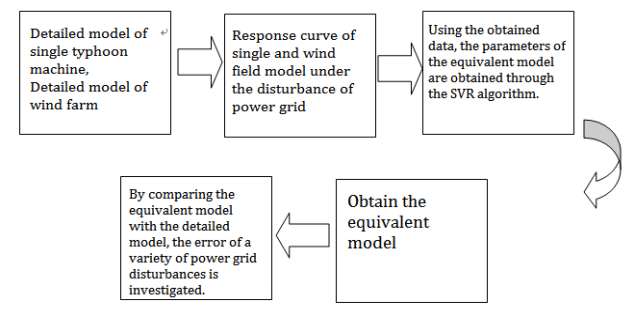

Fig.1 The idea of a special fusion model

\subsection{Feature Fusion Modeling}

In feature fushion modeling, we conbined both methods to get a precise simplified model. Fig2 shows the way to use SVR algorithm to form this model. We extract some features in a turbine motor, and get some emulation data as training set. Then we get all parameters in the simplified model by running all the data in SVR. In this way, we establish a simplified model with significant accuracy because we considered all factors thoroughly.

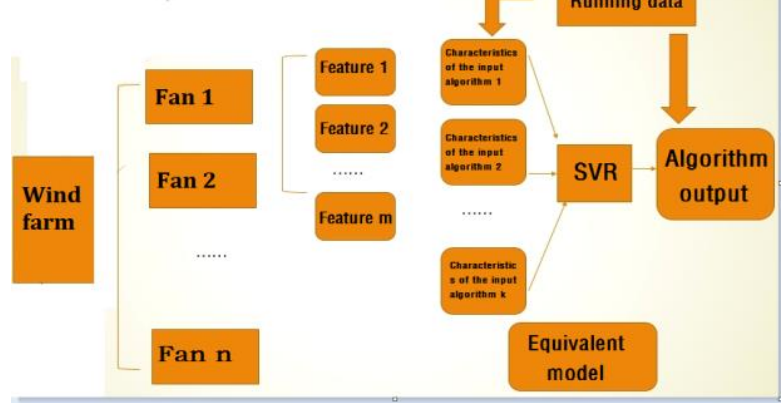

Fig.2 The idea of a special fusion model

\subsection{Support Vector Machine}

The theory of support vector machine (support vector machine, SVM) is created in the late 90's by Corinna Cortes and Vapnik. With the help of libsvm toolkit developed by Professor Lin Zhiren of National Taiwan University, we successfully calculated the parameters in the simplified wind farm model and build it on Cloudpss platform. The general formula show as: 


$$
y=\sum_{\mathrm{i}=1}^{l}\left(a_{\mathrm{i}}-a_{\mathrm{i}}^{*}\right) \mathrm{k}\left(x_{\mathrm{i}}, x\right)+\mathrm{b}
$$

Among them, $y$ is the active power output of the wind farm, $X_{i}$ is the input of the model, the active power output of the wind turbine, $X$ is the support vector, $\mathrm{a}_{\mathrm{i}}, \mathrm{a}_{\mathrm{i}}{ }^{*}$ the coefficients, and $\mathrm{B}$ is the constant term. $\mathrm{K}$ is a kernel function and the core of support vector thought, which determines the parameters and complexity of the model. The common kernel functions include linear kernel function, polynomial kernel function, Gauss kernel function and so on. The formula is shown as follows.

Linear kernel function: $\mathrm{k}\left(\mathrm{x}_{\mathrm{i}}, \mathrm{x}\right)=\mathrm{x}_{\mathrm{i}}{ }^{*} \mathrm{x}$

Polynomial kernel function: $\mathrm{k}\left(\mathrm{x}_{\mathrm{i}}, \mathrm{x}\right)=\left(\mathrm{x}_{\mathrm{i}}{ }^{*} \mathrm{x}+\mathrm{d}\right)^{2}$

Gauss radial kernel function: $\mathrm{k}(\mathrm{xi}, \mathrm{x})=\exp \left(-\left(\mathrm{x}_{\mathrm{i}}-\mathrm{x}\right)^{2} / 2 \sigma\right)$

Considering the physical model of the wind farm, the total active power of the wind farm is equal to the combination of the every operating generator's active power, the linear kernel function is used here.

\section{Simulation of Wind Farm Simplified Model}

Based on the modeling idea of characteristic fusion, the simplified model of the wind farm is built, and the error of the equivalent model is analyzed by running the example of offshore wind farm. Some methods of improving the accuracy is analyzed. The main tasks include:

(1) In detailed model of single motor system, the motor type is direct-driven wind turbine generator. When three-phase short circuit fault happen. We record the response of the single motor in low, medium, high wind speed respectively.

(2)We use the Weibull distribution to calculate the wind speed distribution of the wind farm. Therefore, we know the wind speed at the location of each motor, and calculate the input of mechanical power.

(3) We build a detailed model of the entire wind farm. Combined with the wind distribution model, we record the transient response of the detailed model under the three-phase short circuit falut which happens in the same place in (1)

(4)We use the data collected ablve to calculate the parameters in simplified model with SVR algorithm, and build this linearization model of 3 machines on the Cloudpss simuation platform.

(5)When there are disturbances in the grid or in wind, we examine the error of the simplified model built in (4) to see whether it can produce the accurate response of the wind farm.

\subsection{Single Generator Model}

The single wind turbine of the wind farm is connected to the power grid. The circuit structure is shown in Fig. 3 After the box type transformer is boosted, it enters the $35 \mathrm{kV}$ wind farm bus, and the wind farm is boosted to the $220 \mathrm{kV}$ transmission line at the PCC node, and finally connected to the infinite power grid.The voltage drop of the transmission line is shown in Fig.4.

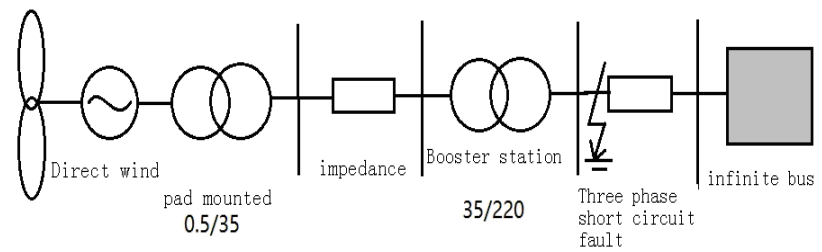

Fig.3 topology structure of wind farm

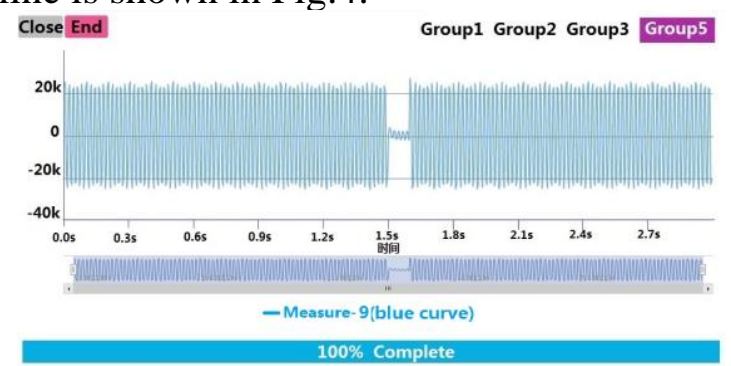

Fig.4 voltage drop 


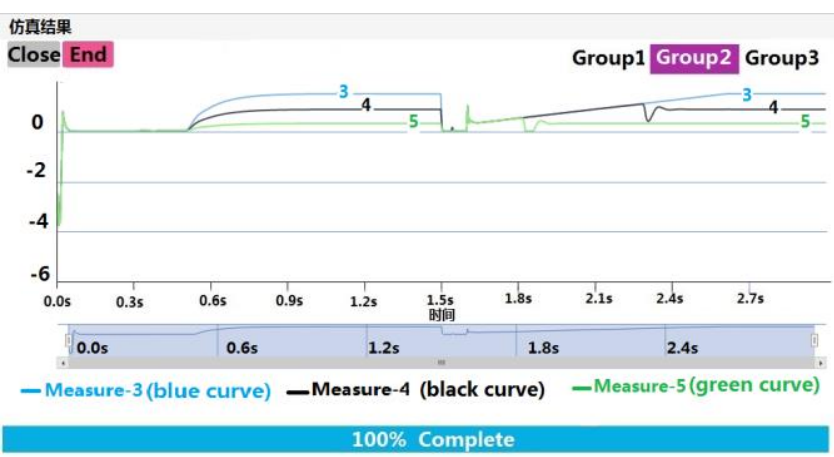

Fig.5 active power curves at low, medium and high wind speeds

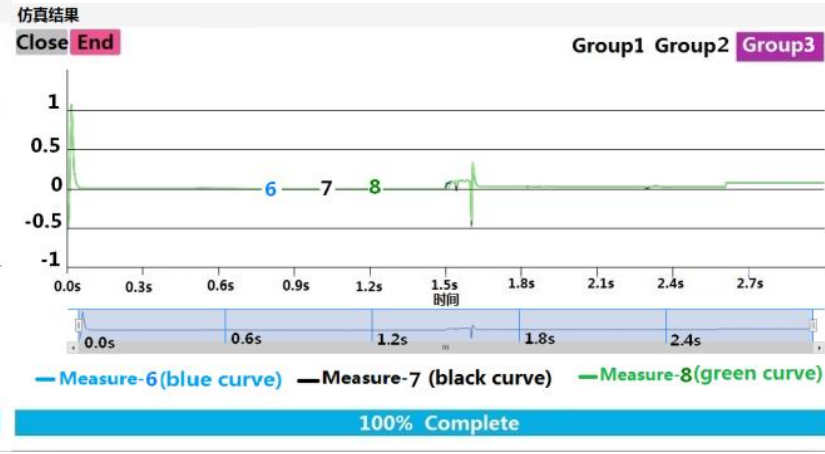

Fig.6reactive power curve of low, medium and high wind speed

In Fig.5, fig.6, The green, black, blue curves respectfully represent the power of generators in $\operatorname{low}(7.6 \mathrm{~m} / \mathrm{s})$, medium $(10.83 \mathrm{~m} / \mathrm{s})$, high $(13 \mathrm{~m} / \mathrm{s})$ wind speed. Fig5 shows the active power curves and Fig6 shows the reactive power curves.

As you can see, the wind speed has great influence on active power output. the transient process of the waveform also showed significant differences, while the three reactive power curves of different wind speed almost overlap. so we mainly focus on getting the accurate active power curve in the simplified model.

\subsection{Wind Speed Model}

Considering the influence of wake effect on the outputs of wind turbines, it is necessary to establish a wind speed model which accords with reality. The distribution of wind speed is influenced by many factors, such as terrain factor, wind turbine arrangement and wind direction. The probability distribution of wind speed is described mainly through the Weibull distribution.

Three parameter Weibull model:

$$
\mathrm{f}(\mathrm{x})=\frac{k}{\lambda}\left(\frac{x-\gamma}{\lambda}\right)^{k-1} \exp \left[-\left(\frac{x-\gamma}{\lambda}\right)^{k}\right]
$$

In the form, $\gamma$ is a shape parameter, $\mathrm{k}$ is the peak of the probability density curve, $\lambda$ is the average wind speed. We often make $\gamma=0$, so a simplified Weibull parameter model with two parameters is obtained.

$$
\mathrm{f}(\mathrm{x})=\frac{k}{\lambda}\left(\frac{x}{\lambda}\right)^{k-1} \exp \left[-\left(\frac{x}{\lambda}\right)^{k}\right]
$$

\subsection{Wind Electric Field Model}

The generators are arranged in $\mathrm{m}$ rows, $\mathrm{n}$ columns. Each generator connects with a transformer in an independent branch, as shown in Fig.7.

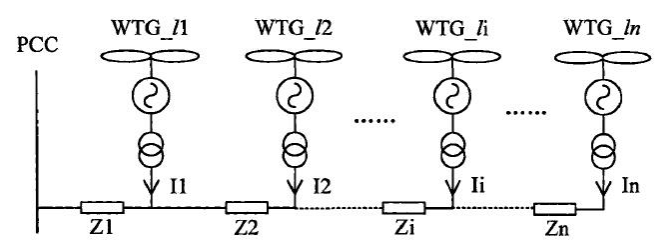

Fig.7 single line model of wind electric field

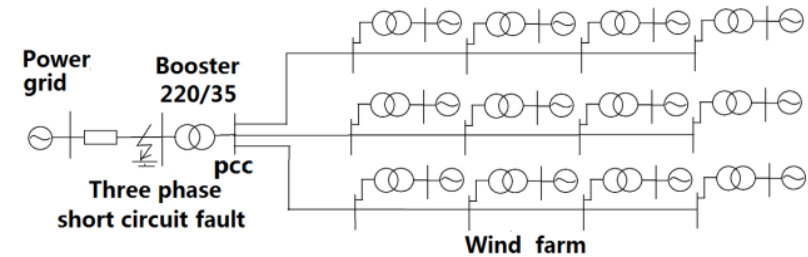

Fig. 8 wind electric field model

Based on the data of an offshore wind farm, we build 12 generators in 3 rows and 4 columns on Cloudpss. The system diagram is shown in Fig.8. Every generator is connected to the $35 \mathrm{kV}$ wind farm bus through a transformer, and the wind farm is boosted into the $220 \mathrm{kV}$ transmission line through PCC node.

The three phase short circuit fault happens in the same place. Fig 9 shows the active power curve(blue curve) and the reactive power curve(black curve) of the entire wind farm. 


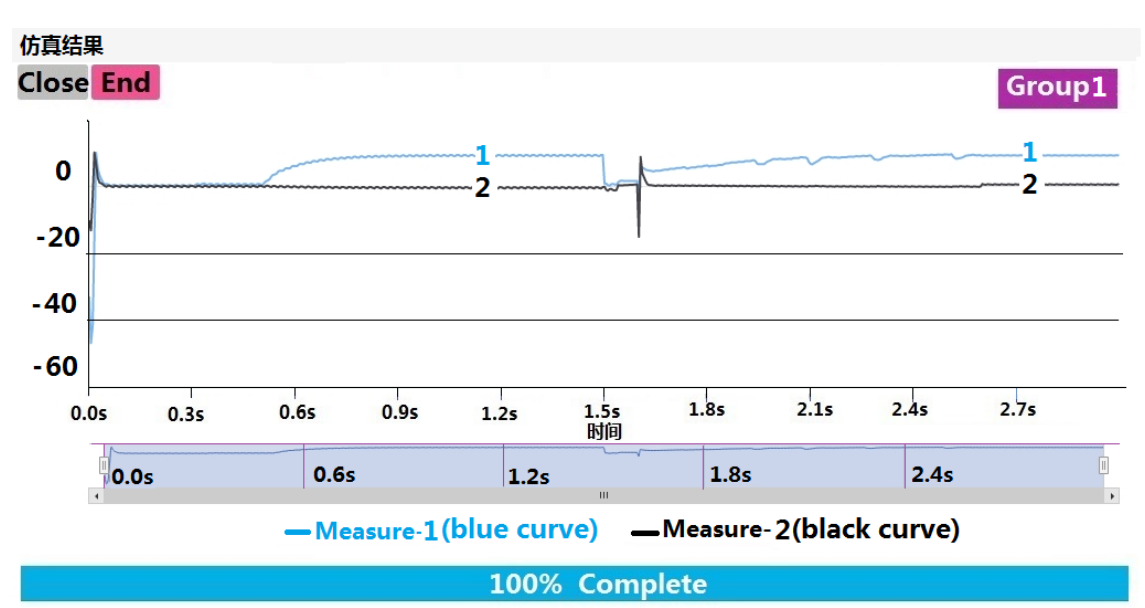

Fig.9 power of wind power

The active power curve data will be used as the output part of the SVR training set. Combined with the single generator model data, the simplified wind farm equivalent model can be obtained.

\subsection{Wind Farm Simplified Model}

We build a charateristic fusion model containing 3 generators operating in different wind speed to calculate the total active power of the wind farm. We use the data of detailed single generator model as inputs of the traing set, and the data of detailed wind farm model as outputs. Considering the total active power of the wind farm is the combination of active power of all generators, we choose linear model as the kernel function and epsilon-SVR as regression algorithm. The results show in Table I :

Table I SVR parameters of the wind farm model

\begin{tabular}{|c|c|c|c|}
\hline $\mathrm{k} 1$ & $\mathrm{k} 2$ & $\mathrm{k} 3$ & Rho(Constant) \\
\hline 0.4241 & 0.4050 & 0.1700 & -0.008849 \\
\hline
\end{tabular}

By the linear kernel function formula: $\mathrm{K}\left(\mathrm{x}_{\mathrm{i}}, \mathrm{x}\right)=\mathrm{x}_{\mathrm{i}}{ }^{*} \mathrm{x}$

The calculation formula for the simplified model of wind farm: $y=K_{1} x[1]+K_{2} x[2]+K_{3} x[3]+b$

The value of $x[1], x[2]$ and $x[3]$ is the active power of low, medium and high speed fan, and the value of $b$ is the opposite number of constant rho. Therefore, based on the linear model formula, $a$ wind farm equivalent model of three wind turbines is built on the Cloudpss platform.

The value of K1, K2 and K3 actually responds to the wind speed distribution of the wind farm, and the sensitivity is very high. For the same training set, the training of different parts of data can lead to large deviation in parameters.

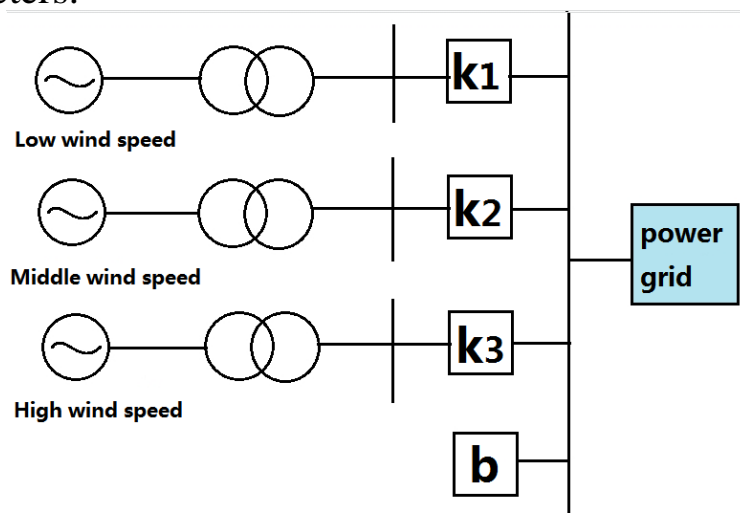

Fig.10 equivalent model of wind field

\subsection{Error Analysis of Simplified Model}

First, we define e to measure the error between simplified model and detailed model. In the transient process, We take $\mathrm{n}$ sample points with the same time distance, and calculate relative error at each point. The value of e equals to the arithmetic mean of $n$ relative errors, which mainly reflects the accuracy of the transient process. 


$$
\mathrm{e}=\frac{1}{n} \sum_{i=1}^{n} \frac{\left|P_{s}-P_{d}\right|}{P_{s}} * 100 \%
$$

We connect the simplified model to a micro grid system, as shown in Fig.11, which contains four motors and four node. The simplified model is incorporated in a node. the three-phase short-circuit fault occurs in the transmission line. Then we exchange the simplified model with the detailed model which has 12 generators to analyse the error of the former.

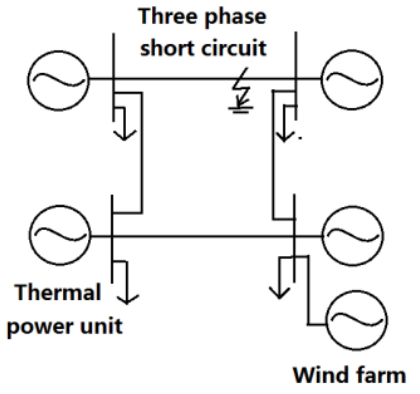

Fig.11 four wind farm system

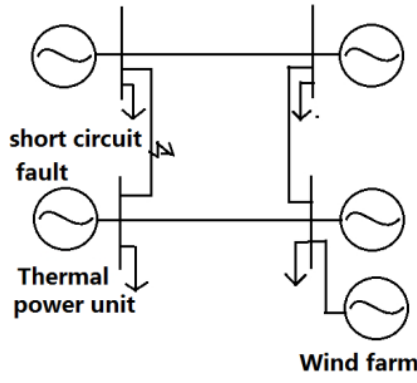

Fig.12 short circuit fault location

The error parameter $\mathrm{e}=3.2 \%$. The static basic error is 0 . The transient process is very good, so it is an accurate simplified model.

When we change the place where three phase short circuit fault happen, e equals to 7\%. It becomes a little larger, because the nonlinear components in complex systems leads to increase. The linear kernel function has its own limitation. In the discussion above, we assume that the wind condition do not change. However, it has some effect on the accuracy of the simplified model.

\subsection{The Influence of Wind Distribution on The Accuracy of The Simplified Model}

The parameters in the simplified model is a reflection of current wind distribution. When the wind becomes stronger or comes from another direction, it will affect the accuracy of the simplified model.

(1) the simplified model under different wind speeds

In the training set, we assume that the top wind speed is $13 \mathrm{~m} / \mathrm{s}$. If the wind increases to $17 \mathrm{~m} / \mathrm{s}$, most generators will be at rated operating state. The input mechanical power of many generators will keep at the rated level which is $1.5 \mathrm{M}$ watt in this case by adjusting the pitch angle.We still use weibull formula to analysis the wind distribution.

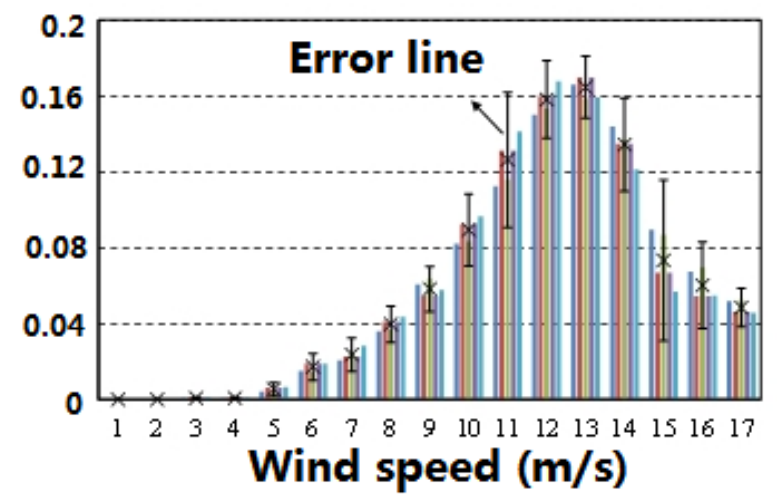

Fig.13 wind velocity distribution

The input mechanical power diagram is obtained, the rated power is $1.5 \mathrm{MW}$.

The value of e is $2.7 \%$. in most areas, the output of detailed model and simplified model are very close, but in 1.5-2.4s the error increases. When we increase the wind speed from 13 to 17, the value sof e are always pretty small(as shown in Table II). So it is still an accurate model.

Table II error of equal value model of wind speed

\begin{tabular}{|c|c|c|c|}
\hline Maximum wind speed & $13 \mathrm{~m} / \mathrm{s}$ & $15 \mathrm{~m} / \mathrm{s}$ & $17 \mathrm{~m} / \mathrm{s}$ \\
\hline Error parameter E & $0.5 \%$ & $1.2 \%$ & $2.7 \%$ \\
\hline
\end{tabular}


(2) the influence of changing the wind direction on the equivalent model

We keeping the wind speed unchanged and assume the wind comes from different directions. The probability distribution is still similar to the Weibull distribution function. The influence of different wind directions on the error of the equivalent model is calculated(Table III).

Table III The influence of the wind direction on the parameters of the equivalent model in table

\begin{tabular}{|c|c|c|c|c|}
\hline & Original model & Wind direction 1 & Wind direction 2 & Wind direction 3 \\
\hline Error parameter E & $0.5 \%$ & $4 \%$ & $8.15 \%$ & $13.1 \%$ \\
\hline K1 & 0.2572 & 0.3375 & 0.4214 & 0.7917 \\
\hline K2 & 0.8472 & 0.4173 & 0.3500 & 0.7044 \\
\hline K3 & 0.6566 & 0.9257 & 0.4476 & 0.2690 \\
\hline
\end{tabular}

When the wind direction changes, the influence on the accuracy of the original simplified model is very significant. As you can see, the values of the parameters $\mathrm{K} 1, \mathrm{~K} 2, \mathrm{~K} 3$ are very sensitive to the change of wind distribution. To guarantee the accuracy of the simplified model,We should work on a program that can recalculate the values of $\mathrm{k} 1, \mathrm{k} 2, \mathrm{k} 3$ automatically when the wind direction changes.

\section{Conclusions}

This paper build a detailed single generator model and detailed wind farm model on Cloudpss platform to get training data. After getting all the parameters by running SVR program, we succesfully build a simplified linear wind farm model. When the wind distribution remains constant, the output of simplified model is very accurate during the grid disturbance.

When the wind condition changes, it could have a significant impact on the accuracy of the simplified wind farm model. If the wind speed increases or decreases, this model is still precise and convinient. However, the changes of wind direction would have a significant impact on the accuracy of the model, so we have to design another program to adjust those parameters automatically.

Characteristics fusion method is a effective way to build a simplified wind farm model. The model is very accurate under most circunstances and has very basic formula. It will shorten the time dramatically in grid stimulation and can help to analysis the transient process.

\section{References}

[1] Lu Qiang Sheng, jade, Chen Ying. Control of [J]. control theory and applications, collaborative giant wind power grid control system 2011, 28 (10): 001491-1495. modeling and Simulation of large scale wind turbines Xiao Jinsong.

[2] Yang Zhe. The main insulation structure of converter transformer for [J]. 800kV DC transmission project [J]. Engineering Technology: full text version, 2016 (12): 00249-00249. wind farm generator active power control simulation research, Zhou Cuiyun.

[3] Shaw Jinsong, Ni Weidou, Jiang Tung. Modeling and Simulation of large wind turbines [J]. solar energy journal, 1997 (2): 117-127.

[4] leaves Jianmin. Research based on [D]. double fedwindfarm fan fusion method characteristic of equivalent model. Beijing: Tsinghua University 2013

[5] Qi Wen. Study on the stability of a large wind farm equivalent model machine combining [D]. Beijing: Beijing Jiaotong University. 2013

[6] Qi Shang Min, Li Fengting, He Shien, et al. Simulation study on fault characteristics of cluster connected to wind farms with low voltage ride through capability. [J]. power system protection and control, 2015, 43 (14): 55-62. 